\title{
Influence of marker models on ankle kinematics in persons with partial foot amputation: An investigation using a mechanical model
}

\author{
Michael Dillon, PhD, BPO(Hons); ${ }^{1-2 *}$ Andrew H. Hansen, PhD; ${ }^{3-4}$ Stefania Fatone, PhD, BPO(Hons) ${ }^{3-4}$ \\ ${ }^{1}$ National Centre for Prosthetics and Orthotics, Division of Allied Health, and ${ }^{2}$ Musculoskeletal Research Centre, \\ La Trobe University, Bundoora, Victoria, Australia; ${ }^{3}$ Northwestern University Prosthetics Research Laboratory and \\ Rehabilitation Engineering Research Program, Department of Physical Medicine and Rehabilitation, Feinberg School \\ of Medicine, Northwestern University, Chicago, IL; ${ }^{4}$ Jesse Brown Department of Veterans Affairs Medical Center, \\ Chicago, IL
}

\begin{abstract}
Previous studies have found that ankle dorsiflexion increases in persons with partial foot amputation walking shod or in "below-ankle" devices compared with walking barefoot. However, a logical biomechanical explanation for these findings has not been offered. The purpose of this project was to determine whether marker location might explain the exaggerated dorsiflexion reported in the literature and, if so, whether more accurate data could be obtained with a different marker set. An articulated mechanical model of the shank and partial foot residuum was constructed with a potentiometer located within the mechanical ankle joint. Ankle angles measured with the calibrated potentiometer were compared with those obtained with both the Helen Hayes marker set and an alternative "residual end" marker set. Results indicated that both marker sets provided accurate and comparable measures at the metatarsophalangeal and transmetatarsal levels. At the Lisfranc level, the Helen Hayes marker set overestimated the ankle angles, primarily because of deformation of the prosthetic forefoot. These results confirm that the choice of kinematic model does influence measurements of ankle motion in persons with partial foot amputation and that the residual end marker set more accurately measures ankle motion at the Lisfranc level.
\end{abstract}

Key words: amputation, biomechanics, gait, kinematics, Lisfranc, marker set, metatarsophalangeal, partial foot, rehabilitation, transmetatarsal.

\section{INTRODUCTION}

A systematic review on the biomechanics of gait in persons with partial foot amputation (PFA) concluded that there was a high level of evidence that PFA affects the temporospatial, external force, kinematic, kinetic, and plantar pressure aspects of PFA gait, but there was less confidence in the evidence regarding exactly how these aspects of gait were affected [1]. Dillon et al. suggested that a number of methodological problems endemic in this body of literature reduced confidence in the evidence [1]. For example, with the exception of two studies [2-3], marker placement was not explicitly described [4-6], which is problematic because marker placement defines the kinematic measurements. Since most authors reported using commercially

Abbreviations: $\mathrm{CMD}=$ coefficient of multiple determination, $\mathrm{HH}=$ Helen Hayes (marker set), MTP = metatarsophalangeal, PFA = partial foot amputation, $\mathrm{RE}=$ residual end (marker set), TMT = transmetatarsal, VA = Department of Veterans Affairs.

*Address all correspondence to Michael Dillon, PhD, BPO(Hons); National Centre for Prosthetics and Orthotics, La Trobe University, Bundoora, Victoria 3086, Australia; +61-3-9479-5889; fax: +61-3-9479-5784.

Email: michael.dillon@latrobe.edu.au

DOI: 10.1682/JRRD.2007.08.0129 
available systems to collect these data [4-6], one can only assume that they followed the standard marker placement procedures required of those systems. However, most of the studies did not describe how certain markers were placed in the presence of a prosthesis or the absence of the forefoot landmarks typically used to locate some of the markers defining the foot local coordinate system. Given these sorts of methodological issues, it is not surprising that the ankle kinematic data vary markedly between investigations. Concerns have been raised regarding the accuracy of these data, especially with respect to discrepancies between barefoot and shod/ device investigations [1].

Previous studies of barefoot walking in PFA suggest that ankle dorsiflexion range is either reduced or similar to that observed in persons without amputation. Garabolsa et al. observed significantly less dorsiflexion of the residuum during gait compared with the sound limb in a group of persons with dysvascular transmetatarsal (TMT) amputation [3]. Boyd et al. investigated groups of persons with dysvascular amputation with either toe, metatarsophalangeal (MTP), or ray amputations, but how many toes or rays were affected in these groups was unclear [5]. No significant differences existed in ankle dorsiflexion between groups of subjects with amputation and a control group of nondisabled subjects who walked more quickly. Unclear descriptions of amputation level [5] make comparison between these studies difficult and walking velocity was either not reported [3] or expressed as a proportion of a laboratory normal database [5]. Hence, accounting for the influence of walking speed between investigations was not possible. Tang et al. reported barefoot kinematics for a group of individuals with "mostly traumatic" TMT amputation as part of an experimental study comparing gait in several conditions, including barefoot, shoe only, or shoe plus prosthesis (insole with carbon fiber footplate) [4]. The dorsiflexion peak observed in the control group was similar to that seen in the PFA barefoot walking condition despite some dorsiflexion bias in the PFA group. Such bias of the kinematic data raises concerns about the modeling and changing of markers between experimental conditions. When the total ankle range from initial plantar flexion peak to dorsiflexion peak is considered, the PFA subjects walking barefoot exhibited reduced angular excursion compared with the control group [4].

In contrast to the relatively normal or reduced ankle dorsiflexion observed during barefoot ambulation in PFA, studies measuring ankle motion in shoe or shoe plus prosthesis conditions suggest that ankle dorsiflexion is increased. Tang et al. reported that for a group of individuals with TMT amputation, the shoe and shoe plus prosthesis conditions allowed significantly greater ankle dorsiflexion during stance compared with walking barefoot or a shod control group [4]. An observational study by Dillon reported a similar pattern of ankle movement in persons with Lisfranc and TMT amputation using either toe fillers, slipper sockets, or shoes stuffed with a variety of materials, but the dorsiflexion range and peak were more normal and comparable with the 95 percent confidence interval of the control group [7].

While previous investigations agree that plantar flexion angle at toe-off and plantar flexion peak are reduced compared with nondisabled controls [2,4,7], when compared with barefoot walking, significant increases in the plantar flexion range were observed simply by fitting a shoe or a shoe plus prosthesis [4]. Such a change in ankle motion is unexpected and would appear to have little mechanical basis.

Considering these results in light of the potential methodological limitations of these studies, one might fairly suggest that uncertainty exists about the capability of "standard" kinematic models, such as the Helen Hayes (HH) marker set [8] to accurately capture motion of the ankle in shod or shod plus device conditions for this group of persons with amputation. Logically, some of the differences observed in the ankle kinematic data between barefoot and shod/device conditions may arise from the effect of motion between the PFA residuum and the shoe/ device (e.g., heel slippage), as has been reported in one investigation [2], or from motion at the "pseudojoint" created between the end of the residuum and devices such as toe fillers. Both scenarios will cause movement between the markers defining the local coordinate system of the foot. The capability of marker placement protocols to account for these unique issues has not been systematically investigated but is necessary for a more accurate understanding of gait in persons with PFA.

The purpose of this investigation was to determine whether the choice of marker location might explain the exaggerated ankle dorsiflexion reported in the literature and, if so, whether more accurate ankle kinematics could be obtained with a different marker set. An articulated mechanical model of the leg with three different length partial foot residua was used to compare the ankle kinematic data derived from a potentiometer (embedded in the ankle of the mechanical model) and conventional marker-based 
gait analysis systems. The marker sets investigated included an HH marker set and a customized "residual end" (RE) marker set. We hypothesized that the customized RE marker set would improve the accuracy of kinematic data in the three levels of PFA compared with the HH marker set and more closely approximate the "gold standard" potentiometer data in spite of any heel slippage or deformation of the forefoot.

\section{METHODS}

The following sections describe the design and calibration of the physical model, the marker sets investigated, and the experimental protocol used to determine the accuracy of each marker set between midstance and late stance, where errors in ankle angles were thought to be most pronounced.

\section{Design of Partial Foot Model}

The partial foot model consisted of shank and foot components separated by a hinge joint representing the ankle (Figure 1). The shank piece was made of wood and

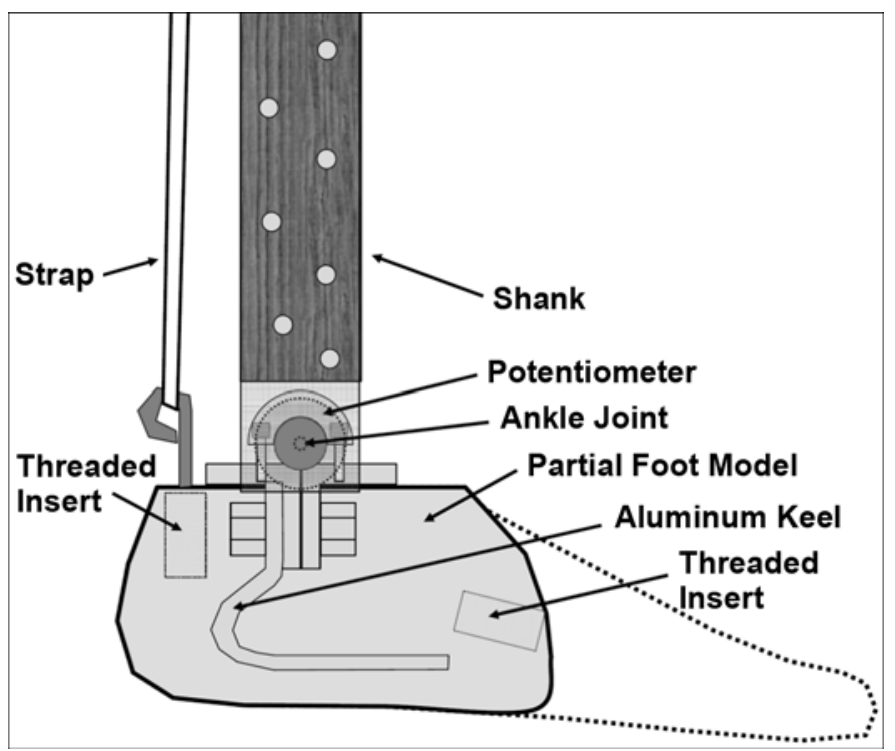

Figure 1.

Schematic of partial foot mechanical model constructed to determine accuracy of various marker sets for measuring ankle kinematics. Dotted line indicates missing portion of foot. Shank and partial foot are separated by hinge joint (ankle joint) with potentiometer in parallel. Partial foot portion has aluminum keel to maintain strength and threaded insert to facilitate lengthening of partial foot residuum. connected to the ankle joint through steel uprights on both sides that were screwed into the wooden piece. The foot sections of the device were made from $\mathrm{PMC}^{\mathrm{TM}}-790$ Shore 90A industrial urethane rubber compound (Smooth-On, Inc; Easton, Pennsylvania) and included several threaded inserts (Figure 1) that allowed for the connection of additional pieces to extend the foot length and mimic different levels of PFA (Figure 2). In this way, the different lengths of the MTP, TMT, and Lisfranc

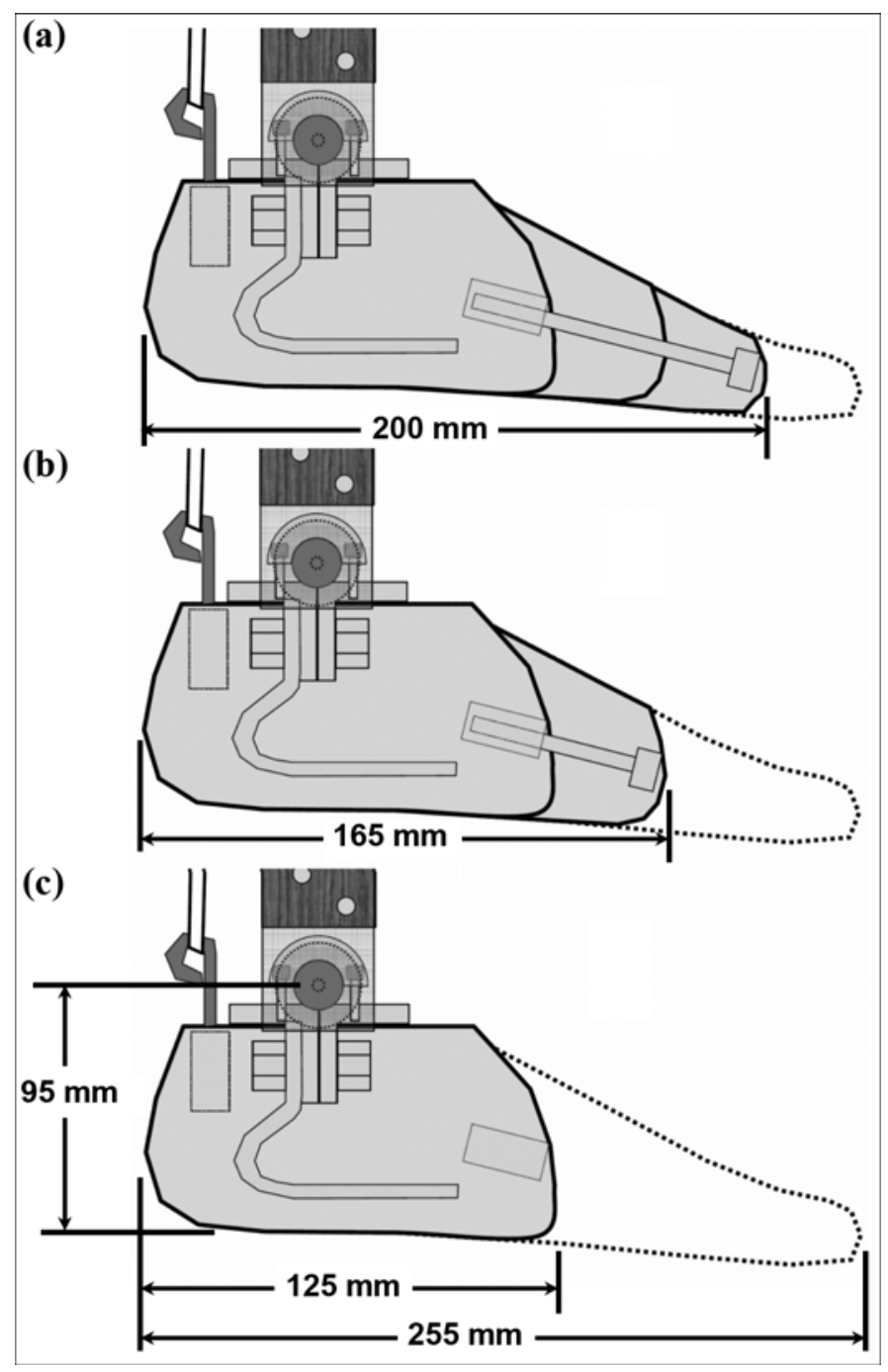

Figure 2.

Schematic of partial foot model with varying partial foot lengths. Three lengths were constructed to represent (a) metatarsophalangeal, (b) transmetatarsal, and (c) Lisfranc amputations based on anthropometric measurements. Source for anthropometric measurements: Dillon MP. Biomechanical models for the analysis of partial foot amputee gait [thesis]. Brisbane (Australia): Queensland University of Technology; 2001. 
residua could be modeled as illustrated in Figure 2(a), 2(b), and 2(c), respectively. The various lengths of the modeled residua were based on anthropometric data reported by Dillon [7].

A rotational potentiometer was placed in-line with the hinge joint and used to measure ankle motion within the device (Figure 1). The housing of the potentiometer was mounted to the shank component, and its shaft was connected to the ankle joint shaft. The ankle joint shaft was rigidly connected to the partial foot piece. Because of this arrangement, rotation of the shank with respect to the partial foot piece turned the shaft of the potentiometer, changing its electrical resistance. The potentiometer was connected to a direct current voltage source on both ends, and the middle pin was connected directly to the motion analysis system (Motion Analysis Corporation; Santa Rosa, California). This direct connection with the motion analysis system allowed synchronization of the potentiometer signal with the marker trajectories being studied. After calibration (described in the "Experimental Protocol" section, p. 571), the potentiometer signal was considered the gold standard measurement of ankle joint motion in this study.

A strap was connected to the posterior superior aspect of the partial foot model and the top of the shank piece (Figure 1). During experiments, the strap was pulled taut when the ankle was slightly plantar flexed. As the shank was rotated over the foot, simulating tibial rotation during stance phase, the strap became more taut, coupling movement of the shank and foot segments.

\section{Marker Sets}

We used two marker sets to calculate ankle angles for comparison with those measured using the potentiometer: a standard $\mathrm{HH}$ marker set and an RE marker set. Ankle joint centers were estimated in both cases by the average of medial and lateral ankle marker positions, and a knee center was estimated by the average of medial and lateral markers located near the top of the shank piece. Ankle markers were placed directly along the ankle shaft of the mechanical model. For the HH marker set, a heel marker and toe marker were placed on the shoe as described by Kadaba et al. [8]. For the RE marker set, markers were placed on the medial and lateral sides of the shoe, proximal to the end of the residuum, and a third marker was placed on the dorsal surface of the residuum, proximal to the end of the residuum (Figure 3).

For the HH marker set, a unit vector was created between the ankle and knee centers and a second unit vector was created between the heel and toe markers. The
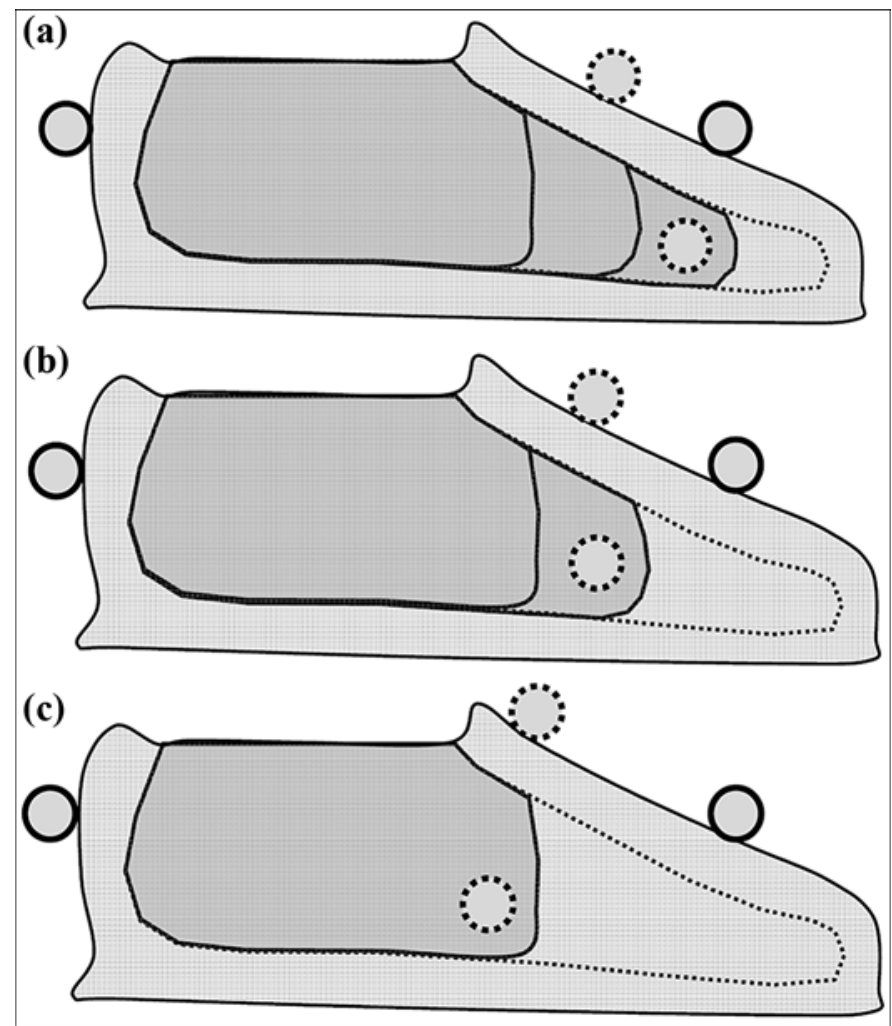

Figure 3.

Schematic of Helen Hayes (solid circles) and residual end (dashed circles) marker sets on (a) metatarsophalangeal, (b) transmetatarsal, and (c) Lisfranc models. Markers for residual end marker set were located proximal to end of residuum irrespective of amputation level. Markers were located on both medial and lateral sides proximal to residual end (one of which is not visible in planar view), and third marker was located on dorsum of residuum, proximal to residual end.

ankle angle for the $\mathrm{HH}$ marker set was calculated from the arcsine of the dot product of these two vectors.

For the RE marker set, the first sample of collected data (while the foot was flat on the floor) was used to create a relationship between the three markers of the RE marker set and the heel and forefoot markers of the HH marker set. A coordinate system was set up by using the three markers of the RE marker set, and vectors between the origin of this coordinate system and the heel and toe markers were created. These vectors were assumed to be static in the local coordinate system and were used to determine virtual heel and virtual forefoot markers for all other samples in the trial. The ankle angle for the RE marker set was then calculated by the same methods as for the HH marker set, except that the virtual heel and virtual forefoot markers were used in the analysis. 


\section{Calibration of Model}

With the model unshod, markers were placed on the posterior aspect of the heel and the dorsal aspect of the residual foot. Markers were also placed at the "ankle" and on the shank as explained in the previous section. With the posterior strap disconnected and the model foot flat on the floor, the ankle was taken through a large range of motion (maximum plantar flexion to maximum dorsiflexion). Marker locations were measured at a frequency of $120 \mathrm{~Hz}$. The potentiometer signal was captured simultaneously with the same motion analysis system but at $960 \mathrm{~Hz}$. This signal was later resampled to $120 \mathrm{~Hz}$ by choosing every eighth point in order to synchronize with the marker data. The potentiometer signal and marker coordinates were then filtered with a bidirectional second order Butterworth filter with a cutoff frequency at $6 \mathrm{~Hz}$ [9]. The ankle angle was calculated as described for the $\mathrm{HH}$ marker set in the previous section. This ankle angle was assumed to be accurate given that the markers used to calculate it were directly placed on the two rigid bodies of the mechanical model. A first order polynomial (i.e., a line) was fitted between the potentiometer signal and the calculated ankle angle. The best-fit coefficients of the polynomial were saved and used to calculate ankle angles from potentiometer signals in the following experiments.

\section{Experimental Protocol}

For each PFA level, a nylon stocking was placed over the foot and the foot was placed into the shoe. The shoe used was a men's size 8W, E.Z. Strider ${ }^{\circledR}$ Walking Shoe (item 75289, Kmart) with a synthetic leather upper, synthetic rubber sole, and two Velcro ${ }^{\circledR}$ straps along the dorsal surface (Figure 4). The insole was removed during the experiments.

In each case, the shoe contained a toe filler necessary to create a snug fit of the partial foot model. Markers were placed on the shoe as shown in Figure 3, with the residual end inside the shoe located by palpation through the outside of the shoe. For each PFA level, two conditions were tested: one in which the Velcro straps were pulled to a "normal" tension and one in which they were closed relatively loosely.

Note that loosening the Velcro straps merely simulated a scenario in which relative movement was likely to occur between either the residuum and shoe or residuum and device, as has been described in previous investigations [10] and reported anecdotally in clinical practice.

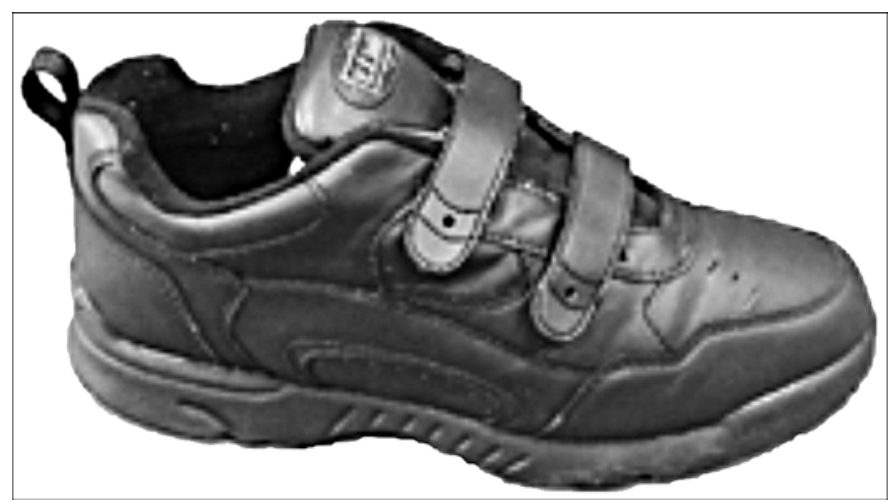

Figure 4.

Men's size 8W, E.Z. Strider ${ }^{\circledR}$ Walking Shoe (item 75289, Kmart) with synthetic leather upper, synthetic rubber sole, and two Velcro ${ }^{\circledR}$ straps along dorsal surface used during investigation. Small holes in ends of Velcro straps allowed tensioning by using spring balance.

The loose condition was accomplished by simply latching the Velcro straps into place without deforming the tongue of the shoe. The normal tension condition was accomplished by pulling the Velcro straps with a spring scale to a force level of $12 \mathrm{lb}(\sim 54 \mathrm{~N})$ and latching them into place at this force level. The spring scale was connected through holes that were punched in the ends of the Velcro straps (Figure 4). The force level used in the normal tension condition was determined by placing a similar shoe on the feet of two nondisabled persons and having them pull the straps (via the spring scale) to a "normal and comfortable" level. In both cases, the force was approximately $12 \mathrm{lb}$.

Data collection with the partial foot model began with the model in a relatively neutral angle. A technician pushed the shank downward and forward to simulate the dorsiflexion that would occur between the midstance and late stance phases of walking. Four trials were taken for each amputation level and strap tightness condition for a total of 24 trials.

\section{Data Analysis}

For all experimental trials with the partial foot model, marker data and data from the potentiometer were filtered with a bidirectional Butterworth filter with a cutoff frequency at $6 \mathrm{~Hz}$ [9]. Ankle angles were calculated for the potentiometer, the HH marker set, and the RE marker set as described earlier. Next, the heel and forefoot markers of the $\mathrm{HH}$ system were examined for the first frame of each trial. Because the trials were always started with the 
foot flat on the floor, the heel and forefoot markers of the $\mathrm{HH}$ system could be used to eliminate any offset dorsiflexion angle. We removed the offset by adding the angle created by the arctangent of the difference in vertical positions of these markers (heel minus forefoot) divided by the absolute difference in horizontal positions of the markers. Lastly, all three ankle angles were assumed to be equal at the beginning of the trials, so offsets were added to the ankle angles determined by the potentiometer and the RE marker set such that they lined up with the ankle angle measured by the $\mathrm{HH}$ marker set.

We compared the ankle angles determined by both marker systems with the potentiometer-determined ankle angle by calculating the coefficient of multiple determination (CMD) as described by Kadaba et al. [11]. A perfect matching of curves would lead to a CMD of 1 . As matching of curves declines, the CMD number is comparatively reduced. This method determined which marker set yielded ankle kinematics that most closely matched the gold standard.

\section{RESULTS}

At both the MTP and TMT levels, good agreement existed between ankle kinematic data calculated by either the RE or HH marker sets and the potentiometer-based measurement system (Figure 5(a)-(b)). However at the TMT level, after about $25^{\circ}$ dorsiflexion, the HH marker set overestimated the ankle joint angles with absolute differences of about $5^{\circ}$ at the largest dorsiflexion angles (Figure 5(b)). At both the MTP and TMT level, the CMD values were comparable between different measurement approaches, highlighting the similarity of the curves (Figure 6).

At the Lisfranc level, good agreement was observed between the three measurement approaches until about $15^{\circ}$ dorsiflexion (Figure 5(c)). After this point, the RE marker set more closely approximated the potentiometer-based measures than did the $\mathrm{HH}$ marker set. The $\mathrm{HH}$ marker set began overestimating ankle joint angles quite dramatically after $15^{\circ}$ dorsiflexion, with small increases in joint angle resulting in substantially overestimated angles. For example, consider the joint angles measured at about frame 100 (Figure 5(c)): the potentiometer and RE marker set describe about $17^{\circ}$ dorsiflexion and the $\mathrm{HH}$ marker set reported angles of about $24^{\circ}$ dorsiflexion. The CMD
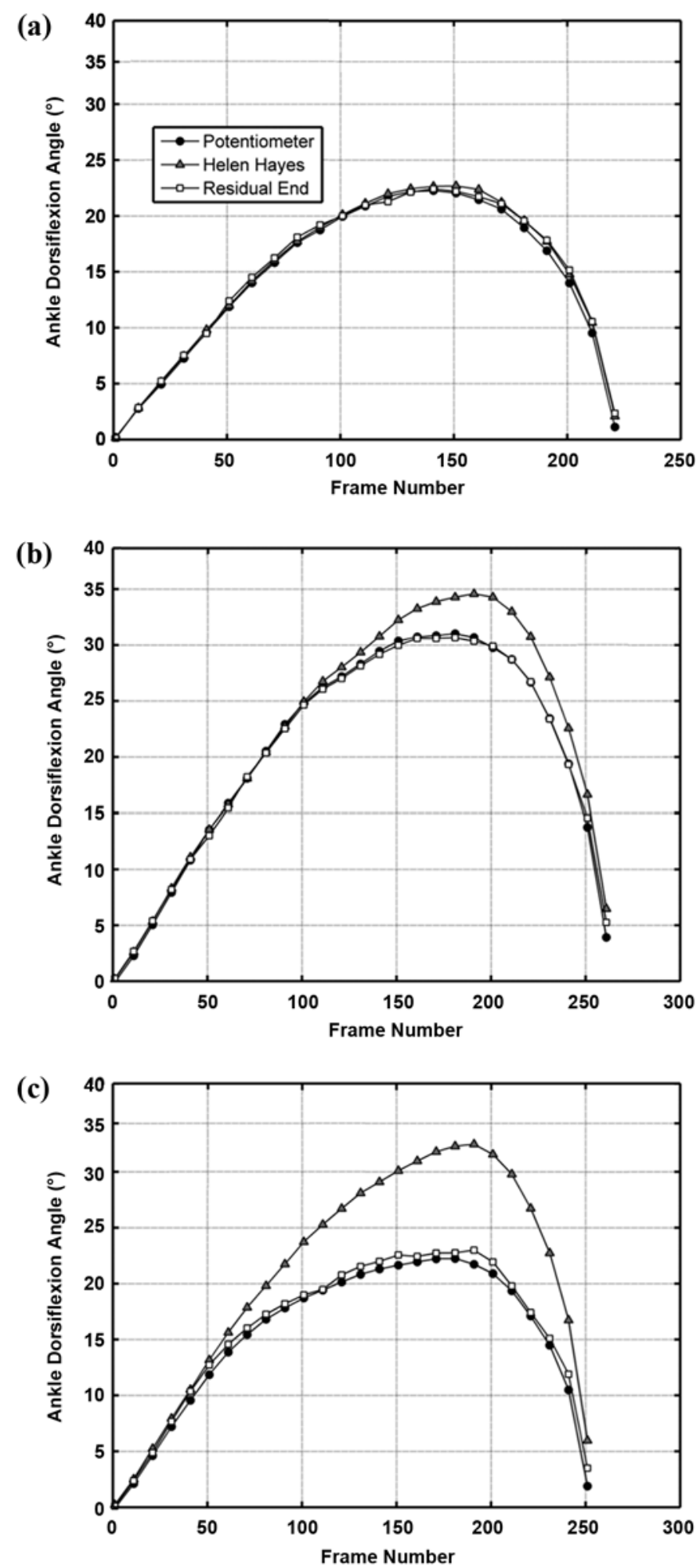

Figure 5.

Ankle joint angles versus time (expressed in frames) collected with potentiometer and both Helen Hayes and residual end marker sets using (a) metatarsophalangeal, (b) transmetatarsal, and (c) Lisfranc models. 
values were significantly reduced with the $\mathrm{HH}$ marker set (Figure 6).

We present the following data to allow the reader to better understand the sources of these errors and appreciate the robustness of the measurement systems to problems such as heel slippage or deformation of the prosthetic forefoot.

When the Velcro straps were loosely closed compared with a more normal strap tension, the following was observed. At the MTP level, the "loose" tension condition did not influence the measurement of ankle kinematics (Figure 7(a)). At the TMT level, good agreement was observed between any of the measurement techniques until about $25^{\circ}$ dorsiflexion, after which the $\mathrm{HH}$ marker set overestimated the ankle joint angles compared with the RE marker set (Figure 7(b)). A similar scenario was observed with the Lisfranc model: ankle angles calculated by the $\mathrm{HH}$ model were overestimated after about $17^{\circ}$ dorsiflexion (Figure 7 (c)).

We evaluated the extent to which deformation of the prosthetic forefoot compromised the rigidity of the foot local coordinate system by comparing ankle joint angles using the $\mathrm{HH}$ marker set and a modification that placed the toe marker of the $\mathrm{HH}$ marker set proximal to the end of the residuum. This modification reduced the overestimated

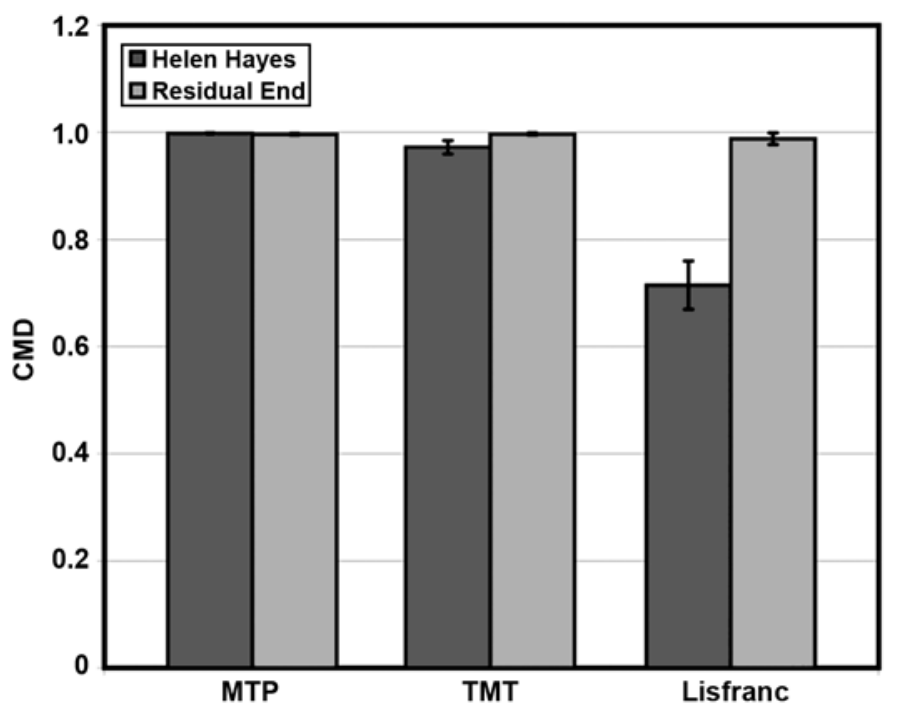

Figure 6.

Coefficient of multiple determination (CMD) for Helen Hayes and residual end marker sets when individually compared with potentiometerderived data in "normal tension" condition. Note that high CMD value approximates 1 at both metatarsophalangeal (MTP) and transmetatarsal (TMT) levels and this value drops significantly for Helen Hayes marker set at Lisfranc level.
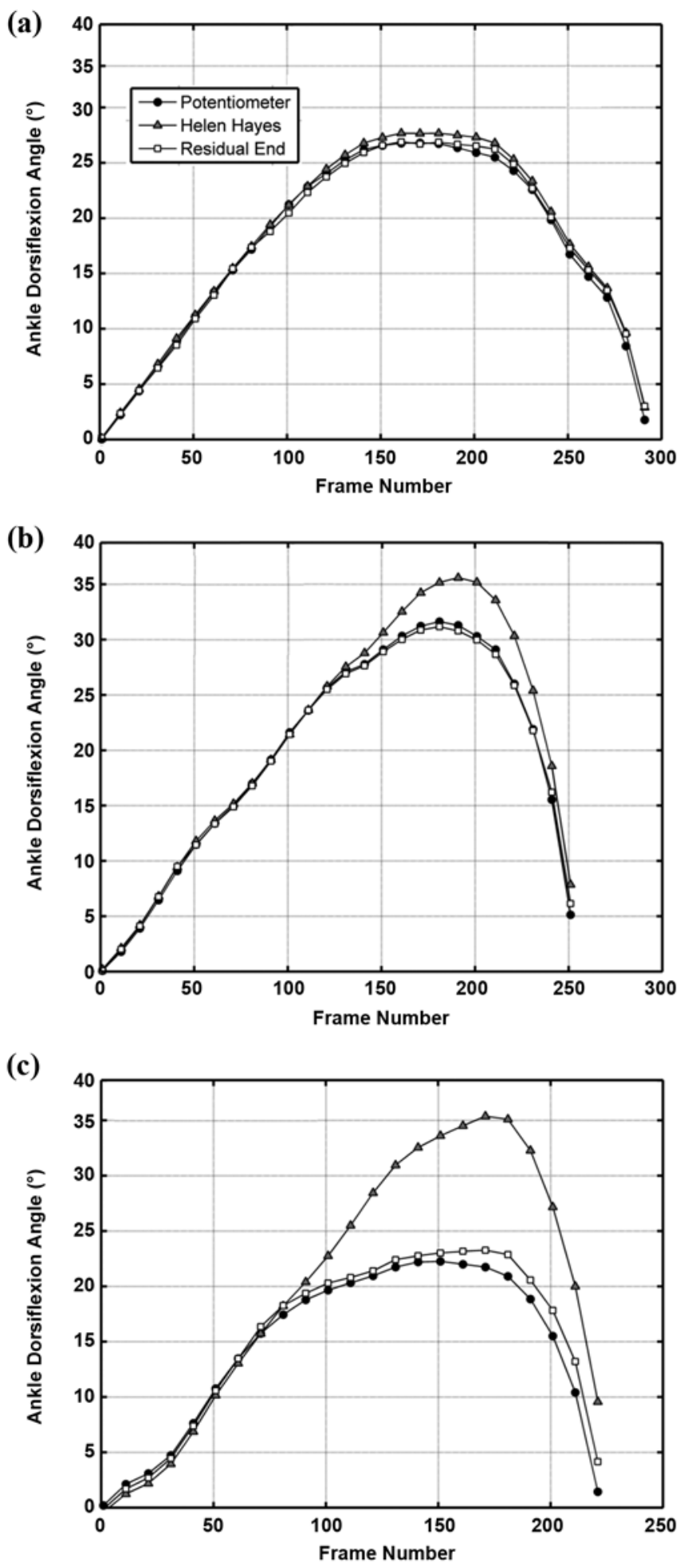

Figure 7.

Ankle joint angles versus time (expressed in frames) collected with potentiometer and both Helen Hayes and residual end marker sets using (a) metatarsophalangeal, (b) transmetatarsal, and (c) Lisfranc models when Velcro ${ }^{\circledR}$ straps were done up loosely to allow heel movement. 
dorsiflexion angles observed with the standard $\mathrm{HH}$ marker set to the point that they were comparable with the potentiometer or RE marker set measurements (Figure 8).

\section{DISCUSSION}

Results from this investigation indicate that the $\mathrm{HH}$ and RE marker sets provide comparable and acceptable measures of ankle kinematics at both the MTP and TMT levels through the measurement range typically associated with normal level walking. However, the published literature does not clarify what constitutes typical ankle dorsiflexion range of motion for PFA, with estimates in shod walking ranging from $10^{\circ}-15^{\circ}$ [7] up to $22^{\circ}-27^{\circ}$ [4], acknowledging what appears to be a $5^{\circ}$ dorsiflexion bias in the latter investigation. As such, using the RE marker set in preference to the $\mathrm{HH}$ marker set may be prudent, because it shows greater accuracy at dorsiflexion angles approaching and in excess of $20^{\circ}$.

At the Lisfranc level, the $\mathrm{HH}$ marker set overestimated ankle angles after about $15^{\circ}$ dorsiflexion and more accurate kinematic data was achieved with the RE marker set.

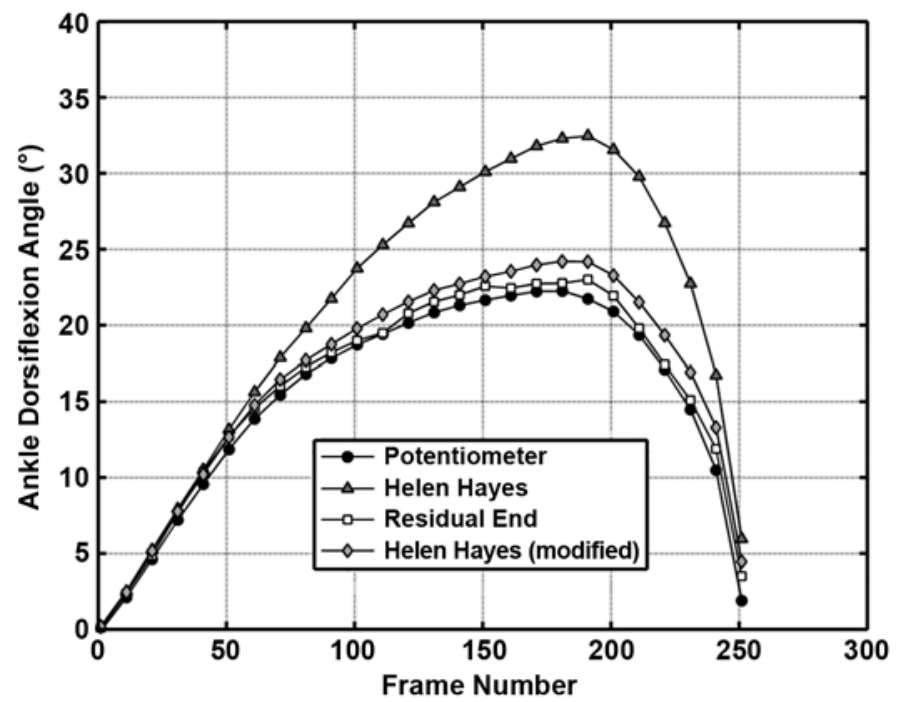

Figure 8.

Ankle joint angles versus time (expressed in frames) collected with potentiometer and both Helen Hayes and residual end marker sets with Lisfranc model. Modified Helen Hayes marker set uses same heel and ankle markers, but toe marker was located more proximally on residuum. Note dramatic improvement in ankle kinematic data that occurred by shifting toe marker.
Errors associated with applying the $\mathrm{HH}$ marker set to study ankle kinematics in PFA can be attributed to either deformation of the forefoot (or in other words, buckling of the shoe at the pseudojoint between the toe filler and residuum) or movement between the shoe and device or shoe and heel of the residuum (Figure 9). In either example, relative motion occurs between markers comprising the foot local coordinate system - thus violating the rigid body assumption upon which kinematic models are based.

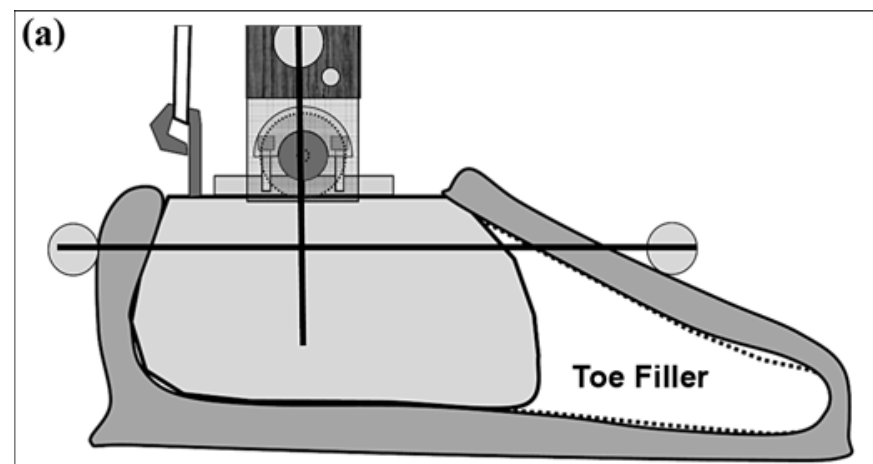

(b)
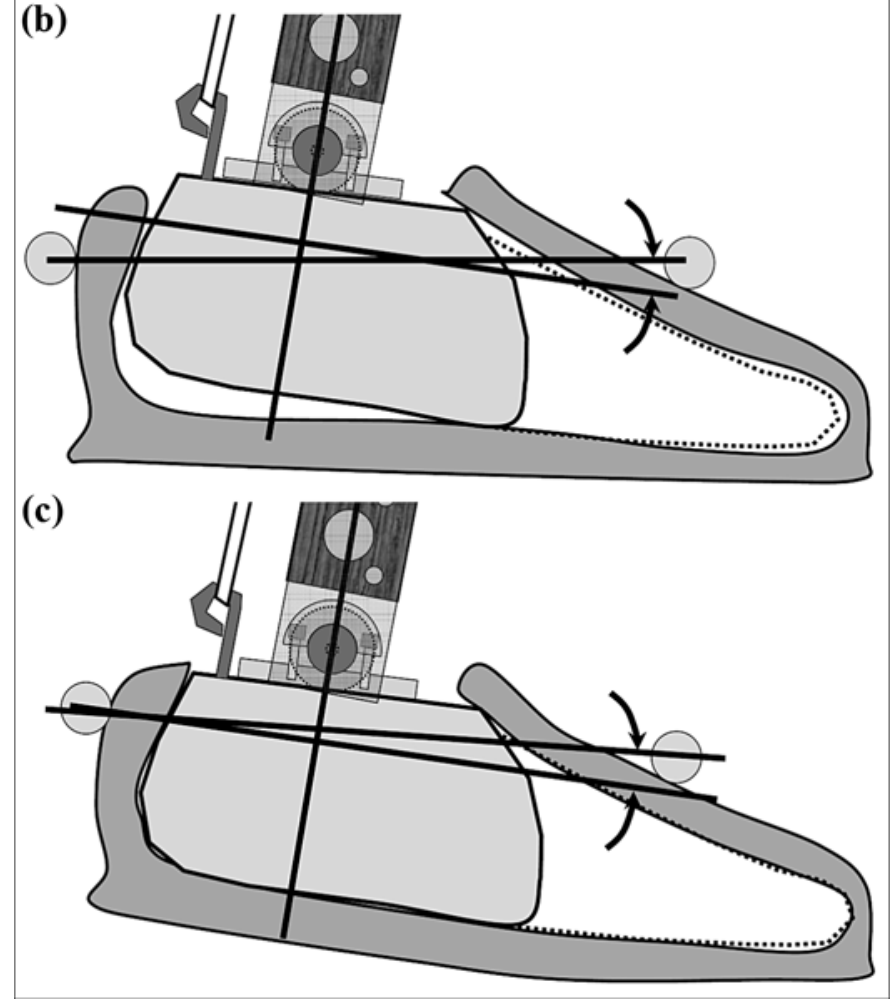

Figure 9.

Anticipated sources of error when the Helen Hayes model is applied to study partial foot amputation. (a) Starting position of tests. (b) Measurement error due to heel slippage. (c) Measurement error due to forefoot bending. 
Should the heel slip within the shoe, the heel marker obviously remains with the shoe, while the ankle marker displaces (Figure 9(b)). In the case of the forefoot deforming, the heel and ankle markers maintain their relationship but the toe marker is displaced relative to the others (Figure 9(c)). In some instances, all these scenarios likely occur at once, making isolation of the individual contribution of each difficult. However, some appreciation of the impact that heel movement or forefoot deformation has on ankle kinematics may be gleaned with different marker-based approaches.

If we used an alternative $\mathrm{HH}$ marker set, in which the toe marker was relocated from its typical position just proximal to the second metatarsal head and moved proximal to the distal residuum, the resulting kinematic data were much improved compared with the standard $\mathrm{HH}$ marker set (Figure 8). This finding illustrates the large effect that errors caused by toe marker displacement have on the measured ankle kinematics and the relatively minor effect that heel slippage will likely have on measurement of these angles. As such, in the absence of a custom model, ensuring that the "toe marker" of an $\mathrm{HH}$ marker set were placed proximal to the residuum end would provide data that more closely approximated that obtained from the potentiometer or RE marker set.

In light of this understanding of the influence of forefoot deformation and heel slippage on kinematic measurements, one might expect that errors in ankle kinematics would be unlikely when the prosthetic forefoot is relatively stiff and the device well suspended. In the case of a toe filler made of a closed cell polyethylene foam in which the forefoot will be relatively compliant or the prosthesis and residuum not well coupled, errors in kinematic measurement are likely. These errors can be attenuated by more appropriate marker sets. In the case of devices with good suspension and relatively stiff forefeet, such as the clamshell prostheses in which the forefoot has been made by cutting down a conventional prosthetic foot, a conventional marker set may yield acceptable results.

Investigators collecting and reporting kinematic data of PFA should consider the appropriateness of the marker set given the type of device being studied, the level of amputation, and the errors that are likely involved. In general, studies that report kinematics need to more explicitly describe the marker sets used and how markers were placed in the absence of forefoot landmarks and in the presence of a prosthesis.
A potential limitation of this work is the capability of the mechanical model to meaningfully replicate what happens in vivo. Although ranges of motion measured with the potentiometer seem to reasonably replicate those seen in vivo, the loads imparted to the shank and foot segments are unlikely to be realistic. The mechanical model is constrained by the loads that it can reasonably support-a potentiometer, for example, will likely fail under the sorts of loading typical of gait. This limitation likely influenced the results. For example, consider the point at which the joint angles from each of the models separate. In Figure 8, that point is at about $15^{\circ}$ dorsiflexion. If more appropriate forces were applied to the shank section and through the strap, we could reasonably expect that the curves would separate at a much smaller dorsiflexion angle, because increased forefoot buckling and heel slippage would be expected. While future studies may investigate in vivo the ankle joint angles calculated by different marker-based approaches, these studies would probably lack a gold standard with which to compare.

\section{CONCLUSIONS}

Results indicated that both the $\mathrm{HH}$ and RE marker sets provided accurate and comparable measures of ankle kinematics at the MTP and TMT levels. At the Lisfranc level, the $\mathrm{HH}$ marker set overestimated the ankle angles primarily because of deformation of the forefoot. The RE marker set accurately measured ankle motion at the Lisfranc level, but where this marker set cannot easily be used, an HH marker set would provide good approximation if the toe marker were placed on the dorsum of the residual foot.

\section{ACKNOWLEDGMENTS}

Data for this project were collected at the Department of Veterans Affairs (VA) Chicago Motion Analysis Research Laboratory of the Jesse Brown VA Medical Center, Chicago, Illinois. We acknowledge the assistance of Rebecca Stine, MS, for acquiring and processing the data; Thomas Most, for helping mold the foot parts; and Craig Heckathorne, MS, for helping instrument the mechanical model.

This material was based on work supported by the National Institute on Disability and Rehabilitation Research 
of the U.S. Department of Education (grant H133E030030) and in part by funding from the Overseas Study Program, La Trobe University.

The opinions contained in this article are those of the grantee and do not necessarily reflect those of the U.S. Department of Education.

The authors have declared that no competing interests exist.

\section{REFERENCES}

1. Dillon MP, Fatone S, Hodge MC. The biomechanics of ambulation after partial foot amputation: A systematic literature review. J Prosthet Orthot 2007;19:(8 Proceedings): P2-P61.

2. Mueller MJ, Salsich GB, Bastian AJ. Differences in the gait characteristics of people with diabetes and transmetatarsal amputation compared with age-matched controls. Gait Posture. 1998;7(3):200-206. [PMID: 10200385]

3. Garbalosa JC, Cavanagh PR, Wu G, Ulbrecht JS, Becker MB, Alexander IJ, Campbell JH. Foot function in diabetic patients after partial amputation. Foot Ankle Int. 1996; 17(1):43-48. [PMID: 8821287]

4. Tang SF, Chen CP, Chen MJ, Chen WP, Leong CP, Chu NK. Transmetatarsal amputation prosthesis with carbonfiber plate: Enhanced gait function. Am J Phys Med Rehabil. 2004;83(2):124-30. [PMID: 14758298]
5. Boyd LA, Rao SS, Burnfield JM, Mulroy SJ, Perry J. Forefoot rocker mechanisms in individuals with partial foot amputation. In: Proceedings of the Clinical Gait and Movement Analysis Society; 1999 Mar 10-13; Dallas, Texas. Clinical Gait and Movement Analysis Society; 1999. p. 144.

6. Wilson EJ, Dillon MP. Restoring centre of pressure excursion using toeoff orthosis in a single partial foot amputee. In: Proceedings of the International Society of Prosthetics and Orthotics, Australian National Members Society; 2005 Nov 3-5; Sydney, Australia. Melbourne (Australia): ISPO ANMS Inc; 2005. p. 31-32.

7. Dillon MP. Biomechanical models for the analysis of partial foot amputee gait [thesis]. Brisbane (Australia): Queensland University of Technology; 2001.

8. Kadaba MP, Ramakrishnan HK, Wootten ME. Measurement of lower extremity kinematics during level walking. J Orthop Res. 1990;8(3):383-92. [PMID: 2324857]

9. Winter DA. Biomechanics and motor control of human movement. 2nd ed. New York (NY): Wiley; 1990.

10. Mueller MJ, Strube MJ. Therapeutic footwear: Enhanced function in people with diabetes and transmetatarsal amputation. Arch Phys Med Rehabil. 1997;78(9):952-56. [PMID: 9305267]

11. Kadaba MP, Ramakrishnan HK, Wootten ME, Gainey J, Gorton G, Cochran GV. Repeatability of kinematic, kinetic, and electromyographic data in normal adult gait. J Orthop Res. 1989;7(6):849-60. [PMID: 2795325]

Submitted for publication August 24, 2007. Accepted in revised form November 20, 2007. 